\title{
Reply to the Response to: Impact of COVID-19 Lockdown on Short-Term Weight Loss in a Single Italian Institution: 1-Year Updated Data
}

\author{
Antonio Vitiello ${ }^{1}\left[\right.$ Giovanna Berardi $^{1} \cdot$ Mario Musella $^{1}$
}

Received: 2 December 2021 / Revised: 2 December 2021 / Accepted: 13 December 2021 / Published online: 16 January 2022

(c) The Author(s), under exclusive licence to Springer Science+Business Media, LLC, part of Springer Nature 2021

\section{Dear Editor,}

As researchers, we always welcome a constructive discussion, but we have to underline that intentions of our previous letter may have been misunderstood.

In our first article [1], we warned the bariatric community about the risks of detrimental effects of lockdown on shortterm weight loss. Afterwards, several articles on the same topic were published [2-5]. In the light of the outcomes of these papers, we updated our study with 1-year results.

In our letter [6], we have enlisted the abovementioned articles [2-5] stressing similarities and differences.

Indeed, the statement "Despite these studies have provided larger samples, they have also included individuals who were submitted to surgery long before the full lockdown" was not specifically referring to one paper [5].

However, once again, we agree with our colleagues: Implementation of telemedicine could be useful to overcome geographical problems and/or to improve healthcare for minorities.

Nevertheless, specific protocols that can provide a safe access to hospitals for critical patients, such as bariatrics, during the current and future pandemics are still mandatory [7].

\section{Declarations}

Ethics Approval All procedures performed in studies involving human participants were in accordance with the ethical standards of the insti-

Antonio Vitiello

antoniovitiello_@hotmail.it

Giovanna Berardi

giovannaberardi88@gmail.com

Mario Musella

mario.musella@unina.it

1 Advanced Biomedical Sciences Department, Naples

"Federico II" University, AOU "Federico II" - Via S. Pansini

5, 80131 Napoli, Italy tutional and/or national research committee and with the 1964 Helsinki declaration and its later amendments or comparable ethical standards.

Informed Consent Informed consent was obtained from all individual participants included in the study.

Conflict of Interest The authors declare no competing interests.

\section{References}

1. Vitiello A, Berardi G, Velotti N, Schiavone V, Musella M. Impact of COVID-19 lockdown on short-term weight loss in a single Italian institution. Obes Surg. 2021;31(7):3365-8. https://doi.org/10. 1007/s11695-021-05343-8.

2. El Moussaoui I, Navez J, El Moussaoui K, et al. Impact of COVID-19 lockdown on short-term results after laparoscopic sleeve gastrectomy. Obes Surg. 2021;31:2614-8. https://doi.org/ 10.1007/s11695-021-05283-3.

3. Conceição E, de Lourdes M, Ramalho S, et al. Eating behaviors and weight outcomes in bariatric surgery patients amidst COVID19. Surg Obes Relat Dis. 2021;17(6):1165-74. https://doi.org/10. 1016/j.soard.2021.02.025.

4. Barranquero AG, Cimpean S, Raglione D, et al. Impact of the COVID-19 pandemic and lockdown on gastric bypass results at 1-year follow-up. OBES SURG. 2021. https://doi.org/10.1007/ s11695-021-05640-2.

5. Pereira X, Romero-Velez G, Skendelas JP, et al. The COVID19 pandemic did not affect target weight loss 1 year post bariatric surgery. Obes Surg. 2021. https://doi.org/10.1007/ s11695-021-05672-8.

6. Vitiello A, Berardi G, Musella M. Impact of COVID-19 lockdown on short-term weight loss in a single Italian institution: 1-year updated data. Obes Surg. 2021. https://doi.org/10.1007/ s11695-021-05759-2.

7. Navarra G, Komaei I, Currò G, et al. Bariatric surgery and the COVID-19 pandemic: SICOB recommendations on how to perform surgery during the outbreak and when to resume the activities in phase 2 of lockdown. Updates Surg. 2020;72(2):259-68. https://doi.org/10.1007/s13304-020-00821-7.

Publisher's Note Springer Nature remains neutral with regard to jurisdictional claims in published maps and institutional affiliations. 